\title{
Periodic flows with global sections
}

\author{
Khadija Ben Rejeb
}

\begin{abstract}
Let $G=\left\{h_{t} \mid t \in \mathbb{R}\right\}$ be a continuous flow on a connected $n$-manifold $M$. The flow $G$ is said to be strongly reversible by an involution $\tau$ if $h_{-t}=\tau h_{t} \tau$ for all $t \in \mathbb{R}$, and it is said to be periodic if $h_{s}=$ identity for some $s \in \mathbb{R}^{*}$. A closed subset $K$ of $M$ is called a global section for $G$ if every orbit $G(x)$ intersects $K$ in exactly one point. In this paper, we study how the two properties "strongly reversible" and "has a global section" are related. In particular, we show that if $G$ is periodic and strongly reversible by a reflection, then $G$ has a global section.
\end{abstract}

\section{Introduction}

Let $X$ be a metric space, and let $G$ be a group of homeomorphisms of $X$. For $x \in X$, the orbit of $x$ under $G$ is

$$
G(x)=\{g(x) \mid g \in G\} ; \text { and }
$$

the isotropy subgroup at $x$ is defined by

$$
G_{x}=\{g \in G \mid g(x)=x\} .
$$

For every subset $A \subseteq X$, we denote by $A / G$ the space of orbits of points of $A$;

$$
A / G=\{G(x) \mid x \in A\} .
$$

The interior of $A$ is denoted by $\operatorname{int}(A)$, and its closure is denoted by $\bar{A}$.

Throughout this paper, an $n$-manifold means a topological manifold of dimension $n$ (that is, a Hausdorff space with a countable basis of open sets, each

Key words and phrases: Periodic flow, strongly reversible, reflection, global section. 2010 Mathematics Subject Classification: 37B05, 57S05, 57S10, 54H20, 37B20. 
homeomorphic to $\mathbb{R}^{n}$ ). A map $\tau: X \longrightarrow X$ is called involution if $\tau$ is a homeomorphism satisfying $\tau^{2}=i d$. The group $G$ is called strongly reversible if there exists an involution $\tau$ of $X$ satisfying

$$
g^{-1}=\tau g \tau, \quad \text { for all } g \in G .
$$

An orbit $G(x)$ is called symmetric (with respect to $\tau$ ) if it is a $\tau$-invariant subset, that is, if $\tau(G(x))=G(x)$.

Reversibility has received a lot of interest in recent years, it plays a role in dynamics and it is related to some problems in physics. A lot of interesting results have been obtained in many works such as [4], [7], [8], [10], [13] and [18].

A subset $K \subset X$ is called a global section (or global cross-section) for $G$ if the following hold:

1. $K$ is closed.

2. Every orbit $G(x)$ intersects $K$ in exactly one point.

A local section (or local cross-section) at a point $p \in X$ is a closed subset $K_{p} \subset X$ satisfying the following conditions.

1. Distinct points of $K_{p}$ lie in distinct orbits.

2. $G_{p}=G_{q}$ for each $q \in K_{p}$.

3. $G\left(K_{p}\right)$ is a neighborhood of $p$.

The concept of sections is a fundamental problem in the theory of dynamical systems. A natural question for cross-sections is existence. Given a group $G$ of homeomorphisms, when $G$ has local or global section? Which necessary and sufficient conditions for the existence of a cross-section?

The existence of a section through a given point shows that local parallelizability of the system is fulfilled. This gives a very good tool for solving many problems as it is possible to describe very precisely the behaviour of a system in a neighborhood of any nonstationary point. There are many works on local and global cross-sections, a brief selection is [1], [6], [9], [14], [15], [16], [17] and [19].

A group $G=\left\{h_{t} \mid t \in \mathbb{R}\right\}$ of homeomorphisms of $X$ is called continuous flow if

$$
\begin{aligned}
& \phi: \quad \mathbb{R} \times X \quad \longrightarrow \quad X \\
& (t, x) \quad \longmapsto \quad h_{t}(x)
\end{aligned}
$$

is a continuous map satisfying:

$$
\begin{aligned}
& \phi(0, x)=x, \text { and } \\
& \phi\left(t, \phi\left(t^{\prime}, x\right)\right)=\phi\left(t+t^{\prime}, x\right) \text { for all } x \in X, t, t^{\prime} \in \mathbb{R} .
\end{aligned}
$$

For $t=0, h_{0}=i d$ is the identity map of $X$. The flow $G$ is called periodic if $h_{s}=i d$ for some $s \in \mathbb{R}_{+}^{*}$. A point $x \in X$ is called periodic if $h_{t}(x)=x$ for some $t \in \mathbb{R}_{+}^{*}$, and it is called stationary if $h_{t}(x)=x$ for all $t \in \mathbb{R}$. 
The existence of local sections was first proved in 1939 by M. Bebutov for flows (Whitney-Bebutov's Theorem [2]).

In this paper, we would like to make comparison between "has a global section" and "strongly reversible". In particular, we study how the two notions are related for periodic flows on connected $n$-manifolds.

The flow $G$ is called parallelizable if there exists a subset $K$ of $X$ such that the restriction

$$
\begin{array}{cccc}
\phi_{\mid \mathbb{R} \times K}: & \mathbb{R} \times K & \longrightarrow & X \\
& (t, x) & \longmapsto & h_{t}(x)
\end{array}
$$

is a homeomorphism. It is easy to show that if $G$ is parallelizable, then the following hold.

1. $G$ has a global section (the subset $K$ ).

2. $G$ has no periodic point.

So, every periodic flow is nonparallelizable. One can ask: Under which conditions periodic flows have global sections?

Let $G$ be a compact abelian Lie group of homeomorphisms of an $n$-manifold $M$ such that $G_{x}=\{i d\}$ for every $x \in M$. Although it is well known that such a group $G$ has local cross-sections everywhere on $M$ (see [16] p. 221), the existence of a global cross-section for $G$ is not guaranteed; a simple example is the flow $G=\left\{h_{t}:\left(z_{1}, z_{2}\right) \longmapsto\left(z_{1} e^{i t}, z_{2} e^{i t}\right) \mid t \in \mathbb{R}\right\}$ on $\mathbb{C} \times \mathbb{C} \backslash\{(0,0)\}$ which is a periodic flow with no global section (Hopf fibration). However, we show the following result.

Theorem 1.1. Let $G$ be a nontrivial periodic flow on a connected n-manifold $M$. Then there exists a finite set of open $G$-invariant subsets $\left\{V_{1}, V_{2}, \ldots, V_{k}\right\}$ of $M$ such that the union $V=\cup_{i=1}^{k} V_{i}$ is dense in $M$, and for every $i$, the following hold.

1. $G_{\mid V_{i}}$ has a global section.

2. $G_{\mid V_{i}}$ is strongly reversible.

If $f$ is a homeomorphism of $X$, we denote by $F i x(f)$ the fixed point set of $f$;

$$
F i x(f)=\{x \in X \mid f(x)=x\} .
$$

Definition 1.2. Let $M$ be a connected $n$-manifold, a map $R: M \longrightarrow M$ is a reflection if the following hold.

1. $R$ is an involution,

2. Fix $(R)$ is an $(n-1)$-submanifold of $M$,

3. $M \backslash F i x(R)$ has two connected components.

When a cross-section exists it need not be locally $(n-1)$-Euclidean (see Example 1.6). Let $G$ be a nontrivial periodic flow on a connected $n$-manifold, in Theorem 1.3 we establish a necessary and sufficient condition for the existence of a global section which is an $(n-1)$-manifold. 
Theorem 1.3. Let $G$ be a nontrivial periodic flow on a connected $n$-manifold M. Then the following are equivalent:

1. $G$ has a global section $K$ which is an $(n-1)$-manifold.

2. $G$ is strongly reversible by an involution $\tau$ such that Fix $(\tau) \backslash F$ has two connected components $A$ and $B$ and each of $A \cup F$ and $B \cup F$ is a closed (n-1)-manifold.

In the following theorem, we prove that for periodic flows on connected $n$-manifolds, "strongly reversible by a reflection" implies "have global section".

Theorem 1.4. Let $G$ be a nontrivial periodic flow on a connected n-manifold $M$. If $G$ is strongly reversible by a reflection $R$, then $G$ has a global section.

Remark 1.5. 1) From Theorem 1.4, we can see that the flow

$$
G=\left\{h_{t}:\left(z_{1}, z_{2}\right) \longmapsto\left(z_{1} e^{i t}, z_{2} e^{i t}\right) \mid t \in \mathbb{R}\right\}
$$

on $\mathbb{C} \times \mathbb{C}$ is an example of a periodic flow that cannot be strongly reversible by a reflection since $G$ has no global section. However $G$ is strongly reversible by the involution $\left.\tau:\left(z_{1}, z_{2}\right) \longmapsto\left(\overline{z_{1}}, \overline{z_{2}}\right)\right)$; where $\operatorname{Fix}(\tau) \cong \mathbb{C}$.

2 ) For $n \geq 4$, a periodic flow on $\mathbb{R}^{n}$ which is strongly reversible by a reflection $R$ need not have a global section which is a manifold (Example 1.6).

Example 1.6. Let $B$ be the Bing's dog bone space. It is well known that $B$ is not a manifold, and that $B \times \mathbb{R}$ is homeomorphic to $\mathbb{R}^{4}$. We define a flow $G$ on $B \times \mathbb{R}^{2}$ by

$$
h_{t}:(b, x) \longmapsto\left(b, x e^{i t}\right) .
$$

Clearly, $G$ is a periodic flow on $B \times \mathbb{R}^{2}$ which is homeomorphic to $\mathbb{R}^{5}$. Moreover, $G$ is strongly reversible by $R:\left(b, x_{1}, x_{2}\right) \longmapsto\left(b,-x_{1}, x_{2}\right)$ which is a reflection with $F i x(R)=B \times \mathbb{R} \cong \mathbb{R}^{4}$. The fixed point set $F i x(G)=B \times\{0\}$ divides $F i x(R)$ into two connected components $B \times \mathbb{R}_{+}^{*}$ and $B \times \mathbb{R}_{-}^{*}$, and $B \times \mathbb{R}_{+}$is a global section for $G$, however, $B \times \mathbb{R}_{+}$is not a manifold.

In studying periodic flows on connected manifolds, we will need the following theorem.

Theorem 1.7. ([5], Theorem 9.5, p. 157) If $G$ is a compact Lie group acting effectively on a connected n-manifold $M$, then the set $M^{G}=\{x \in M \mid g(x)=$ $x$ for all $g \in G\}$ is nowhere dense.

The paper is organized as follows. In Section 2, we study compact groups with global sections, and we investigate their properties. In Section 3, we consider the particular case of periodic flows on connected $n$-manifolds; where we begin by proving some general results of such flows and we prove Theorem 1.1. In Subsection 3.1, we study periodic flows with global sections, and in Subsection 3.2, we 
study periodic flows which are strongly reversible, then, we prove Theorem 1.3 in Subsection 3.3. Subsection 3.4 is about Theorem 1.4.

\section{Compact groups with global sections}

Let $G$ be a compact abelian group of homeomorphisms of a metric space $M$. In the following proposition we give a necessary condition for the existence of a global section for $G$.

Proposition 2.1. Let $G$ be a compact abelian group of homeomorphisms of a metric space $M$. If $G$ has a global section $K$ then $G$ is strongly reversible by an involution $\tau$.

Proof. Since $K$ is a global section for $G$, for every $x \in M$, the orbit $G(x)$ intersects $K$ in exactly one point, it follows that $M \subset G(K)$. Conversely, clearly $G(K) \subset M$. Then $M=G(K)$. Define a map $\tau$ on $M$ as follows:

$$
\begin{aligned}
& \tau: \quad G(K) \quad \longrightarrow \quad G(K) \\
& g(x) \longmapsto g^{-1}(x)
\end{aligned}
$$

We will show that $\tau$ is an involution and $G$ is strongly reversible by $\tau$. The map $\tau$ is well defined because if $g(x)=g^{\prime}\left(x^{\prime}\right)$ in $G(K)$ then $x=x^{\prime}$ since $K$ is a global section, so $g(x)=g^{\prime}(x)$ which implies that $g^{-1}(x)=g^{\prime-1}(x)$.

For showing the continuity of $\tau$ let $\left(y_{n}\right)_{n}=\left(g_{n}\left(x_{n}\right)\right)_{n}$ be a sequence in $G(K)$ converging to $y=g(x) \in G(K)$. Let $B=\left\{y_{n}, n \geq 0\right\} \cup\{y\}$. By compactness of $G$ and $B, G(B)$ is compact. Let $\left(g_{n_{k}}^{-1}\left(x_{n_{k}}\right)\right)_{k}$ be a subsequence of $\left(g_{n}^{-1}\left(x_{n}\right)\right)$, converging to some point $b$. Let $g_{n_{\varphi(k)}}$ be a subsequence of $\left(g_{n_{k}}\right)_{k}$ converging to some element $g_{0} \in G$. It follows that $x_{n_{\varphi(k)}}$ converges to $g_{0}(b) \in K$ since $K$ is closed and $g_{n_{\varphi(k)}}\left(x_{n_{\varphi(k)}}\right) \longrightarrow g_{0}\left(g_{0}(b)\right)=g(x)$, which implies that $g_{0}(b)=x$ since $K$ is a global section, and so $g_{0}^{-1}(x)=g^{-1}(x)=b$. It follows that $\left(g_{n}^{-1}\left(x_{n}\right)\right)_{n}$ converges to $g^{-1}(x)$.

It is easy to see that $\tau^{2}=i d$, then $\tau$ is bijective and $\tau^{-1}=\tau$ is continuous. So $\tau$ is a homeomorphism. Thus $\tau$ is an involution.

Now, we show that for every $f \in G, \tau f \tau=f^{-1}$. Let $y=g(x) \in G(K)$, then $\tau f \tau(y)=\tau f \tau(g(x))=\tau f\left(g^{-1}(x)\right)=f^{-1}(y)$ since $G$ is abelian. We conclude that $G$ is strongly reversible by $\tau$.

Example 2.2. The converse implication in the above Proposition is not true; that is, a strongly reversible compact abelian group need not have global section. Consider for example the action of the circle group $G$ on $\mathbb{C} \times \mathbb{C}$ given in Remark 1.5.(1);

$$
G=\left\{h_{t}:\left(z_{1}, z_{2}\right) \longmapsto\left(z_{1} e^{i t}, z_{2} e^{i t}\right) \mid t \in \mathbb{R}\right\}
$$


So, clearly $G$ is a compact abelian group and is strongly reversible by $\tau:\left(z_{1}, z_{2}\right) \longmapsto$ $\left(\overline{z_{1}}, \overline{z_{2}}\right)$, however $G$ has no global section.

Proposition 2.3. Let $G$ be a compact group of homeomorphisms of a metric space $M$ having a global section $K$. If $G_{x}=\{i d\}$ for every $x \in M$, then $G \times K$ is homeomorphic to $M$.

Proof. Since $K$ is a global section, $M=G(K)$. Consider the map $\phi$ defined by

$$
\begin{aligned}
\phi: \quad G \times K & \longrightarrow G(K) \\
(g, x) & \longmapsto g(x)
\end{aligned}
$$

We will show that $\phi$ is a homeomorphism. It is easy to see that $\phi$ is well defined and continuous. Injectivity of $\phi$ follows from the fact that $K$ is a global section and $G_{x}=\{i d\}$ for every $x \in M$. To show that $\phi^{-1}$ is continuous, let $g_{n}\left(x_{n}\right) \longrightarrow g(x)$ in $G(K)$. Since $G$ is compact, let $\left(g_{n_{k}}\right)_{k}$ be a subsequence of $\left(g_{n}\right)$ converging to $g_{0} \in G$. Then $x_{n_{k}} \longrightarrow g_{0}^{-1}(g(x))=x_{0} \in K$ since $K$ is closed. Which implies that $g(x)=g_{0}\left(x_{0}\right)$, so $x=x_{0}$ and $g=g_{0}$ since $G_{x}=\{i d\}$. We deduce that $g_{n} \longrightarrow g$ and $x_{n} \longrightarrow x$. Thus $\phi^{-1}$ is continuous, and $\phi$ is a homeomorphism.

In the following Lemma, we show that if a group $G$ of homeomorphisms of a metric space $M$ has a global section $K$, then $K$ is homeomorphic to the orbit space $M / G$.

Lemma 2.4. Let $G$ be a group of homeomorphisms of a metric space $M$. If $G$ has a global section $K$, then the following hold.

1. The restriction of the orbit map $\pi: M \longrightarrow M / G$ to $K$ given by

$$
\begin{array}{cccc}
\pi_{\mid K}: & K & \longrightarrow & K / G=M / G \\
x & \longmapsto & G(x)
\end{array}
$$

is a homeomorphism.

2. If $M$ is connected, then $K$ is connected.

Proof. (1) It is easy to see that the orbit map

$$
\begin{aligned}
\pi: M & \longrightarrow M / G \\
x & \longmapsto G(x)
\end{aligned}
$$

is continuous and open. Since $K$ is a global section, then $K / G=\{G(x) \mid x \in K\}=$ $M / G$ and the restriction

$$
\begin{aligned}
\pi_{\mid K}: \quad K & \longrightarrow M / G \\
x & \longmapsto G(x)
\end{aligned}
$$


is a bijection. Moreover, $\pi_{\mid K}$ is continuous since $\pi$ is. For showing the continuity of $\left(\pi_{\mid K}\right)^{-1}$, let $F$ be a closed subset of $K$, we will show that $\pi(F)$ is closed in $M / G$. Since $K$ is closed in $M$, then $F$ is closed in $M$, then $\pi(F)$ is closed in $M / G$. We conclude that $\pi_{\mid K}$ is a homeomorphism.

(2) Assume that $K$ is not connected, then there exist two open subsets $V_{1}$ and $V_{2}$ in $K$ such that $V_{1} \cap V_{2}=\varnothing$ and $K=V_{1} \cup V_{2}$ which implies that

$$
M=G(K)=G\left(V_{1}\right) \cup G\left(V_{2}\right) . \quad(*)
$$

Since $V_{1}$ is open in $K$, then by Item $1, \pi_{\mid K}\left(V_{1}\right)=\pi\left(V_{1}\right)$ is open in $M / G$ and since $\pi$ is continuous, then $\pi^{-1}\left(\pi\left(V_{1}\right)\right)=G\left(V_{1}\right)$ is open in $M$, in the same way, $G\left(V_{2}\right)$ is open in $M$. Moreover, $G\left(V_{1}\right) \cap G\left(V_{2}\right)=\varnothing$ since $V_{1} \cap V_{2}=\varnothing$ and $V_{1}, V_{2} \subset K$, where $K$ is a global section. So, equality $(*)$ contradicts the fact that $M$ is connected. Thus $K$ is connected.

In the following Proposition, we show that if $G$ is a compact Lie group of homeomorphisms of an $n$-manifold $M$ such that $G_{x}=\{i d\}$ for every $x$, then $M$ is covered by a finite set of $G$-invariant open subsets such that the restriction of $G$ to each of them has a global section.

Proposition 2.5. Let $G$ be a compact Lie group of homeomorphisms of an $n$-manifold $M$ such that for every $x \in M, G_{x}=\{i d\}$. Then there exists a finite set $\left\{U_{1}, U_{2}, \ldots, U_{k}\right\}$ of subsets of $M$ such that for every $1 \leq i \leq k, U_{i}$ is contained in a local cross-section $K_{i}, G\left(U_{i}\right)$ is open in $M$, and

$$
M=G\left(U_{1}\right) \cup \ldots \cup G\left(U_{k}\right) .
$$

Proof. By Lemma 2.4.(1), the orbit map $\pi: M \longrightarrow M / G$ is a homeomorphism on local cross-sections. From the Proof of Theorem 4.2 of [15], there exists a collection $F=\left\{A_{i}\right\}_{i}$ of closed subsets $A_{i}$ of $M / G$ satisfying the following conditions:

1. $M / G=\cup_{i} \operatorname{int}\left(A_{i}\right)$.

2. There exists a finite collection $\left\{L_{1}, L_{2}, \ldots, L_{k}\right\}$ of closed subsets $L_{i}$ of $M / G$ such that:

a) for every $1 \leq i \leq k$, there exists a subcollection $F_{i}=\left\{A_{i_{j}}\right\}_{j}$ of $F$ which is a covering of $L_{i} ; L_{i}=\cup_{j} A_{i_{j}}$;

b) for every $1 \leq i \leq k, L_{i}=\pi\left(K_{i}\right)$; where $K_{i}$ is a closed local section in $M$, and c) $\cup_{i=1}^{k} F_{i}=F$.

By (1) and (2).(c), we have $M / G=\cup_{i=1}^{k}\left(\cup_{j} \operatorname{int}\left(A_{i_{j}}\right)\right)$. For every $1 \leq i \leq k, U_{i}^{\prime}=$ $\cup_{j} \operatorname{int}\left(A_{i_{j}}\right) \subset L_{i}$ is open in $M / G$, then $\pi^{-1}\left(U_{i}^{\prime}\right)$ is open in $M$, by (2).(b), $U_{i}^{\prime}=$ $\pi\left(U_{i}\right)$; where $U_{i} \subset K_{i}$, then $\pi^{-1}\left(U_{i}^{\prime}\right)=G\left(U_{i}\right)$ is open in $M$, and $M=\pi^{-1}(M / G)=$ $\cup_{i=1}^{k} \pi^{-1}\left(U_{i}^{\prime}\right)=G\left(U_{1}\right) \cup \ldots \cup G\left(U_{k}\right)$. 


\section{Periodic flows on connected manifolds}

Let $G$ be a continuous flow on a metric space $X$. A point $x \in X$ is said to be stationary if

$$
h_{t}(x)=x, \quad \text { for all } t \in \mathbb{R},
$$

and it is said to be periodic if

$$
h_{t}(x)=x, \quad \text { for some } t>0 .
$$

If $x \in X$ is periodic, but nonstationary, then there is $T>0$ such that $T$ is the smallest positive real satisfying $h_{T}(x)=x$ (see Theorem 2.12 of [6]). This real $T$ is called the period of $x$, and for every real $t>0$ satisfying $h_{t}(x)=x$, there is an integer $n \geq 1$ such that $t=n T$. The flow $G$ is called periodic of period $s>0$ if $h_{s}=i d$ and for every $0<t<s, h_{t} \neq i d$.

Proposition 3.1. Let $G$ be a continuous flow on a metric space $X$. Then the following hold.

1. Given any $\varepsilon>0$, the set $A_{\varepsilon}=\left\{x \in X \mid h_{t}(x)=x\right.$ for some $\left.0<t \leq \varepsilon\right\}$ is closed.

2. For $x \in X$, the following are equivalent.

a) $x$ is stationary.

b) There is a sequence $\left(x_{n}\right)_{n}$ in $X$ and a sequence $\left(t_{n}\right)$ in $\mathbb{R}_{+}^{*}$ such that $h_{t_{n}}\left(x_{n}\right)=x_{n}$ for every $n, t_{n} \longrightarrow 0$, and $x_{n} \longrightarrow x$.

Proof. 1) See Theorem 2.14 of [6].

2) $(a) \Longrightarrow(b)$ Assume that $x$ is stationary, then for every integer $n>0, h_{\frac{1}{n}}(x)=x$. Then $t_{n}=\frac{1}{n} \longrightarrow 0$, and $x_{n}=x \longrightarrow x$.

$(b) \Longrightarrow(a)$ Assume (b) is true. Let $\varepsilon>0$. Since $t_{n} \longrightarrow 0$, there exists an integer $n_{0}>0$ such that for every $n \geq n_{0}, t_{n} \leq \varepsilon$, then $x_{n} \in A_{\varepsilon}$ for every $n \geq n_{0}$, and since $A_{\varepsilon}$ is closed by Item 1 , then $x \in A_{\varepsilon}$; that is, $h_{t_{\varepsilon}}(x)=x$ for some $0<t_{\varepsilon} \leq \varepsilon$. Then $x$ is periodic. Assume that $x$ is nonstationary, then $x$ is with period $T \neq 0$ and $t_{\varepsilon}=k_{\varepsilon} T$ for some $k_{\varepsilon} \in \mathbb{N}^{*}$. Then $T=\frac{t_{\varepsilon}}{k_{\varepsilon}} \leq t_{\varepsilon} \leq \varepsilon$, when $\varepsilon \longrightarrow 0$, we obtain $T=0$; a contradiction, so $x$ is stationary.

In the remainder of this section, we take $G=\left\{h_{t} \mid t \in \mathbb{R}\right\}$ to be a nontrivial continuous flow on $M$ which is periodic with period $s \in \mathbb{R}_{+}^{*}$. We shall make use of the following notations:

$$
\begin{gathered}
F=\{x \in M \mid x \text { is stationary }\} ; \\
L=\{x \in M \mid x \text { is with period } 0<t<s\} ; \\
H=\{x \in M \mid x \text { is with period } s\} .
\end{gathered}
$$


It is easy to see that $M=L \cup H \cup F$. We denote by $N=L \cup H=M \backslash F$ the set of nonstationary points of $M$. Clearly the set $F$ is closed as intersection of closed sets (see Section 1 for the definition of a stationary point).

Lemma 3.2. Let $G$ be a periodic flow of period $s$ on a metric space $M$. Then the subset $H$ is open.

Proof. For showing that $H$ is open, we will show that $L \cup F$ is closed. Let $\left(x_{n}\right)_{n}$ be a sequence in $L \cup F$ such that $x_{n} \longrightarrow x$. We show that $x \in L \cup F$. For every $x_{n} \in L \cup F$, there exists $0<t_{n}<s$ such that $h_{t_{n}}\left(x_{n}\right)=x_{n}$. Since $\left(t_{n}\right) \subset[0, s]$ compact, then we can assume that $t_{n} \longrightarrow t_{0} \in[0, s]$. If $t_{0}=0$, then $x \in F$ by Proposition 3.1.(2). If $t_{0}=s$, then $\left(s-t_{n}\right) \longrightarrow 0$ and $h_{s-t_{n}}\left(x_{n}\right)=x_{n}$ for every integer $n$ since $h_{s}=i d$. So $x \in F$ by Proposition 3.1.(2). If $0<t_{0}<s$, then $h_{t_{0}}(x)=x$, and either $x \in F$ or $x$ is with period $0<T<s$, thus $x \in L$. We conclude that $L \cup F$ is closed.

Lemma 3.3. Let $G$ be a periodic flow of period $s$ on a metric space $M$. Then for every subset $V \subset L$, the following hold.

1. For every $x_{0} \in V$, there exists $\varepsilon>0$ such that every point $x$ in $B\left(x_{0}, \varepsilon\right) \cap V$ is with period $T_{x} \geq T_{0}$; where $T_{0}$ is the period of $x_{0}$.

2. There exists a nonempty open subset $U$ in $V$ on which all points have the same period.

Proof. (1) Assume that statement (1) is not true, then there exists $x_{0} \in V$ such that for every $\varepsilon>0$, there exists $x_{\varepsilon} \in B\left(x_{0}, \varepsilon\right) \cap V$ such that $T_{\varepsilon}<T_{0}$; where $T_{\varepsilon}$ is the period of $x_{\varepsilon}$. Then for every $n \in \mathbb{N}^{*}$, there exists $x_{n} \in B\left(x_{0}, \frac{1}{n}\right) \cap V$ such that $T_{n}<T_{0}$; where $T_{n}$ is the period of $x_{n}$. We know that $\left(T_{n}\right)$ has a convergent subsequence, we may assume that $\left(T_{n}\right)$ converges. But $\left(T_{n}\right) \subset\left\{\frac{s}{k} \mid k \in \mathbb{N}^{*}\right\}$ since $G$ is with period $s$, then when $n \longrightarrow+\infty$, either $T_{n} \longrightarrow 0$ or $T_{n} \longrightarrow \frac{s}{k_{0}}$. If $T_{n} \longrightarrow 0$, since $x_{n} \longrightarrow x_{0}$ and $h_{T_{n}}\left(x_{n}\right)=x_{n}$ for every $n$, then by Proposition 3.1.(2), $x_{0}$ is stationary which is a contradiction since $x_{0} \in V \subset L$. Then $T_{n} \longrightarrow \frac{s}{k_{0}}$. So there exists a positive integer $n_{0}$ such that for all $n \geq n_{0}, T_{n}=\frac{s}{k_{0}}$. When $n \longrightarrow+\infty$, we obtain $h_{\frac{s}{k_{0}}}\left(x_{0}\right)=x_{0}$, which implies that $\frac{s}{k_{0}}=p T_{0}$ for some $p \in \mathbb{N}^{*}$ but $\frac{s}{k_{0}} \leq T_{0}$, then $p=1$ and $\frac{s}{k_{0}}=T_{0}$ which contradicts the fact that $T_{n}<T_{0}$ for all $n$. We conclude that (1) is true.

(2) Let $x_{0} \in V$. By Item 1 there exists $\varepsilon>0$ such that every point $x$ in $B\left(x_{0}, \varepsilon\right) \cap$ $V$ is with period $T_{x} \geq T_{0}$. Let $T_{0}=\frac{s}{k_{0}}$ and let $T_{x}=\frac{s}{k_{x}} \geq \frac{s}{k_{0}}$. Then $k_{x} \leq k_{0}$, so $\left\{T_{x} \mid x \in\right.$ $\left.B\left(x_{0}, \varepsilon\right) \cap V\right\} \subset\left\{\frac{s}{k} \mid 2 \leq k \leq k_{0}\right\}$. It follows that $B\left(x_{0}, \varepsilon\right) \cap V=\cup_{k=2}^{k_{0}} A_{k}$; where $A_{k}=$ $\left\{x \in B\left(x_{0}, \varepsilon\right) \cap V \mid x\right.$ is with period $\left.\frac{s}{k}\right\}$, we have $A_{i} \cap A_{j}=\varnothing$ for all $i \neq j$ in $\left\{2, \ldots, k_{0}\right\}$. Since $B\left(x_{0}, \varepsilon\right) \cap V$ is open in $V$, then for some $2 \leq k \leq k_{0}, \operatorname{int}\left(A_{k}\right) \neq \varnothing$; where $\operatorname{int}\left(A_{k}\right)$ is the interior of $A_{k}$ in $V$. Thus $U=\operatorname{int}\left(A_{k}\right)$ is a nonempty open subset in $V$ on which all points have the same period $\frac{s}{k}$. 
Proposition 3.4. Let $G$ be a nontrivial periodic flow of period s on a connected n-manifold $M$. Then the following hold.

1. $G$ is a compact connected Lie group of dimension 1 , and $N=M \backslash F$ is connected.

2. The subset $H$ is open and everywhere dense in $M$.

Proof. (1) If $F=\varnothing$, then clearly $N=M$ is connected. Now, assume that $F \neq \varnothing$. We will show that $N=M \backslash F$ is connected. Suppose that $M \backslash F$ is nonconnected, then there exist two open subsets $U_{1}$ and $U_{2}$ such that $U_{1} \cap U_{2}=\varnothing$ and $M \backslash F=$ $U_{1} \cup U_{2}$. Since $G$ is connected, then for every $t \in \mathbb{R}, h_{t}\left(U_{1}\right)=U_{1}$ and $h_{t}\left(U_{2}\right)=U_{2}$. Let $G^{\prime}=\left\{h_{t}^{\prime} \mid t \in \mathbb{R}\right\}$ be the flow on $M$ defined by

$$
h_{t}^{\prime}(x)= \begin{cases}h_{t}(x), & \text { if } x \in U_{1} . \\ x, & \text { if } x \in F \cup U_{2}\end{cases}
$$

Clearly $G^{\prime}$ is a compact Lie group since $G$ is, and since $G^{\prime}=i d$ on $U_{2}$, then $G^{\prime}=$ $\{i d\}$ on $M$ by Theorem 1.7 , so $G=\{i d\}$ on $U_{1}$, and hence $G=\{i d\}$ on $M$, which contradicts the fact that $G$ is nontrivial. Thus $M \backslash F$ is connected.

(2) Assume that $\operatorname{int}(L \cup F) \neq \varnothing$. Let $V=\operatorname{int}(L \cup F) \backslash F$. Then $V$ is open in $M$. We will show that $V \neq \varnothing$. Assume that $V=\varnothing$, then $\varnothing \neq \operatorname{int}(L \cup F)=F \cap \operatorname{int}(L \cup F) \subset$ $F$ and $G=\{i d\}$ on $\operatorname{int}(L \cup F)$, since $G$ is a compact Lie group by Item 1 , then by Theorem 1.7 $G$ is trivial, which is absurd. Then $V \neq \varnothing$. By Lemma 3.3.(2), there exists a nonempty open subset $U$ of $V$ on which all points have the same period $T=\frac{s}{k},(k \geq 2)$. Then $h_{T}=i d$ on $U$. But $h_{T}$ is periodic of period $k$, then by Theorem 1.7, $h_{T}=i d$ everywhere on $M$, which is impossible since $T<s$. We conclude that $\operatorname{int}(L \cup F)=\varnothing$, equivalently, $\bar{H}=M$. Moreover, $H$ is open by Lemma 3.2.

Proof of Theorem 1.1. By Proposition 3.4.(1), $G$ is a compact Lie group. Since $H$ is an open (Lemma 3.2), $G$-invariant subset of $M$, then the restriction $G_{\mid H}$ is a compact Lie group. For every $x \in H, G_{x}=\{i d\}$, then by Proposition 2.5, there exists a finite set $\left\{U_{1}, \ldots, U_{k}\right\}$ of subsets of $H$ such that for every $i, U_{i}$ is contained in a local cross-section $K_{i}$ in $H, G\left(U_{i}\right)$ is open in $H$ and $H=\bigcup_{i=1}^{k} G\left(U_{i}\right)$. By Proposition 3.4.(2), $\bar{H}=M$. For every $i$, we will show that the subset $U_{i}$ is closed in $G\left(U_{i}\right)$. Let $\left(x_{n}\right)$ be a sequence in $U_{i}$ converging to some point $x$ in $G\left(U_{i}\right)$, we must show that $x \in U_{i}$. We have $x=h_{t}\left(x_{0}\right)$ for some real $t$ and some $x_{0} \in U_{i}$, on the other hand $U_{i} \subset K_{i}$ and since $K_{i}$ is closed, $x \in K_{i} \cap G\left(U_{i}\right)$. Therefore, $x$ and $x_{0}$ are in $K_{i}$ and are in the same orbit, so by the definition of a local section, $x=x_{0} \in U_{i}$. Now, we can easily see that $U_{i}$ is a global section for $G_{\mid G\left(U_{i}\right)}$, and $G_{\mid G\left(U_{i}\right)}$ is strongly reversible by Proposition 2.1 . 


\subsection{Periodic flows with global sections}

Let $G$ be a periodic flow of period $s$ on a connected $n$-manifold $M$ having a global section $K$. Recall that $G$ is compact (Proposition 3.4). In this subsection, we like to prove some properties of $G$. We begin by showing, in the following Lemma, that $G$ is strongly reversible, and that the fixed point set of $h_{\frac{s}{2}}$ coincides with the set $F$ of stationary points of $G$.

Lemma 3.5. Let $G$ be a periodic flow of period $s$ on a connected $n$-manifold M. If $G$ has a global section $K$, then the following hold.

1. $\overline{K \cap H}=K$.

2. $G$ is strongly reversible by the involution

$$
\begin{aligned}
\tau: \quad G(K)=M & \longrightarrow G(K) \\
h_{t}(x) & \longmapsto h_{-t}(x) .
\end{aligned}
$$

and $F i x(\tau)=K \cup\left\{h_{\frac{T}{2}}(x) \mid x \in K \backslash F\right.$ and $x$ is with period $\left.T\right\}$.

3. $F i x\left(h_{\frac{s}{2}}\right)=F$.

Proof. (1) Since $M=\bar{H}$ by Proposition 3.4.(2), and $M=G(K)$, then $M=$ $\overline{G(K) \cap H}=\overline{G(K \cap H)}$ since $H$ is $G$-invariant. By compactness of $G$, we obtain

$$
M=G(\overline{K \cap H}) .
$$

Since $K$ is closed, $\overline{K \cap H} \subset K$. Assume that $K \neq \overline{K \cap H}$, then there exists $y \in K$ such that $y \notin \overline{K \cap H}$. By $(*), y=h_{t}(x)$ for some $x \in \overline{K \cap H} \subset K$, then $y=x$ (since $K$ is a global section); which is a contradiction. Thus $K=\overline{K \cap H}$.

(2) The flow $G$ is strongly reversible by the involution $\tau$ (see Proof of Proposition 2.1). It is easy to see that if $F \neq \varnothing$ then $F \subset K$ since $M=G(K)$. Let $y=h_{t}(x) \in$ $G(K)$ such that $\tau(y)=y$, then $h_{-t}(x)=h_{t}(x)$, equivalently, $h_{2 t}(x)=x$. Then either $x=y \in F$ or $2 t=n T$ for some $n \in \mathbb{N}^{*}$; where $T$ is the period of $x$, equivalently, $t=n \frac{T}{2}$. If $n=2 p$ is even, then $t=p T$, so $y=h_{p T}(x)=x \in K$. If $n=2 p+1$ is odd, then $t=p T+\frac{T}{2}$ and $y=h_{\frac{T}{2}}(x)$. Thus $F i x(\tau) \subset K \cup\left\{h_{\frac{T}{2}}(x) \mid x \in K \backslash F\right.$ and $x$ is with period $\left.T\right\}$. The converse inclusion, is easy to see. So $F i x(\tau)=A \cup B \cup F$; where $K=A \cup F$ and $B=\left\{h_{\frac{T}{2}}(x) \mid x \in A\right.$ and $x$ is with period $\left.T\right\}$.

(3) Let $y \in F i x\left(h_{\frac{s}{2}}\right)$. Since $M=G(K)$, then $y=h_{t}(x)$ for some $x \in K$, and $h_{\frac{s}{2}}(x)=x$. Assume that $x \notin F$; that is, $x \in K \backslash F$. Since $A=K \backslash F$ is open in $K$, then $V_{x}=A \cap h_{\frac{s}{2}}(A)$ is a nonempty open subset in $K$ containing $x$ and satisfying $h_{\frac{s}{2}}\left(V_{x}\right)=V_{x}$. It follows by Lemma 2.4.(1) that $G\left(V_{x}\right)$ is open in $M$. Since $\bar{H}=M$, then $G\left(V_{x}\right) \cap H \neq \varnothing$, which implies that $V_{x} \cap H \neq \varnothing$ since $H$ is $G$-invariant. Let $z \in\left(V_{x} \cap H\right) \subset V_{x} \subset A$, then $z$ is with period $s$ and $h_{\frac{s}{2}}(z) \in B \cap V_{x} \subset B \cap A$; which is impossible since $A \cap B=\varnothing$. Therefore $x \in F$, and $F i x\left(h_{\frac{s}{2}}\right) \subset F$. The converse inclusion is clear, thus Fix $\left(h_{\frac{s}{2}}\right)=F$. 
In the following proposition "dim" means the topological dimension. The topological dimension of a topological space $E$ is defined inductively as follows: the empty set is assigned dimension -1 , and $E$ is said to be $n$-dimensional at a point $p$ if $n$ is the least number for which there are arbitrarily small neighborhoods of $p$ whose boundaries in $M$ all have dimension $<n$. The space $E$ has topological dimension $n$ if its dimension at all of its points is $\leq n$ but is equal to $n$ at one point at least.

Proposition 3.6. Let $G$ be a nontrivial periodic flow of period s on a connected $n$-manifold $M$. If $G$ has a global section $K$, then $G$ is strongly reversible by the involution

$$
\begin{aligned}
\tau: \quad G(K) & \longrightarrow G(K) \\
h_{t}(x) & \longmapsto
\end{aligned}
$$

satisfying the following properties

1. F divides Fix $(\tau)$ into two connected components $K \backslash F$ and $h_{\frac{s}{2}}(K \backslash F)$.

2. $\operatorname{dim} K=(n-1)=\operatorname{dim} F i x(\tau)$.

Proof. (1) The subset $M \backslash F$ is connected by Proposition 3.4.(1), then by Lemma 2.4.(2), $K \backslash F$ is connected. By Lemma 3.5, Fix $(\tau)=A \cup B \cup F$; where $A=$ $K \backslash F$, and $B=\left\{h_{\frac{T}{2}}(x) \mid x \in K \backslash F\right.$ and $x$ is with period $\left.T\right\}$. Since $G$ is with period $s$, then $h_{\frac{s}{2}}(F i x(\tau))^{\frac{T}{2}}=F i x(\tau)=h_{\frac{s}{2}}(A) \cup h_{\frac{s}{2}}(B) \cup F$. We will show that $A \cap h_{\frac{s}{2}}(A)=\varnothing$. If not, there exists $y \in A \cap h_{\frac{s}{2}}(A)$, then $y=h_{\frac{s}{2}}(x)$ for some $x \in A$, then $y=x$ since $A \subset K$, and $h_{\frac{s}{2}}(x)=x$. Since $F i x\left(h_{\frac{s}{2}}\right)=F$ by Lemma 3.5.(3), then $x \in F$, which is a contradiction. So, $A \cap h_{\frac{s}{2}}(A)=\varnothing$. It follows that $h_{\frac{s}{2}}(A) \subset B$. Since $A=\left\{h_{\frac{T}{2}}(x) \mid x \in\right.$ $B$ and $x$ is with period $T\}$ and $h_{\frac{s}{2}}(B)=\left\{h_{\frac{T}{2}}\left(h_{\frac{s}{2}}(x)\right) \mid x \in A\right.$ and $x$ is with period $\left.T\right\}$, then $h_{\frac{s}{2}}(B) \subset A$. Therefore $h_{\frac{s}{2}}(A)=B$, equivalently $h_{\frac{s}{2}}(B)=A$. Since $B=(F i x(\tau)) \backslash$ $K$ is open in $F i x(\tau)$, then $B$ is open in $(A \cup B)$. Which implies that $A$ is closed in $(A \cup B)$ since $A \cap B=\varnothing$. Since $h_{\frac{s}{2}}: A \cup B \longrightarrow A \cup B$ is a homeomorphism, then each of $A$ and $B=h_{\frac{s}{2}}(A)$ is open and closed in $A \cup B$. Moreover, each of $A$ and $B$ is connected since $A$ is connected. Thus Fix $(\tau) \backslash F=A \cup B$ has two connected components $A$ and $B$; that is, $F$ divides $F i x(\tau)$ into two connected components $A=K \backslash F$ and $B=h_{\frac{s}{2}}(A)$.

(2) Since $H$ is $G$-invariant, then $H=G(K \cap H)$ and $K \cap H$ is a global section for the restriction $G_{\mid H}$. Moreover, $G_{x}=\{i d\}$, for every $x \in K \cap H$, then the map

$$
\begin{array}{rlr}
\phi: \quad G \times(K \cap H) & \longrightarrow & H \\
\left(h_{t}, x\right) & \longmapsto & h_{t}(x)
\end{array}
$$

is a homeomorphism by Proposition 2.3. Since $H$ is open in $M$, then $\operatorname{dim} H=$ $n=\operatorname{dim}(G \times(K \cap H)) \leq \operatorname{dim} G+\operatorname{dim}(K \cap H)$. On the other hand, since $G$ is compact, it is well known that $\operatorname{dim}(G \times(K \cap H))>\max \{\operatorname{dim} G, \operatorname{dim}(K \cap H)\}$ (see [11]). 
Since $\operatorname{dim} G=1$ by Proposition 3.4.(1), then $\operatorname{dim}(K \cap H)<n \leq 1+\operatorname{dim}(K \cap H)$. So, $\operatorname{dim}(K \cap H)=(n-1) \leq \operatorname{dim} K$. If $\operatorname{dim} K=n$, then $K$ contains a nonempty open subset $U$ of $M$. Therefore, $U \cap H$ is open in $M$ and $\operatorname{dim}(U \cap H)=n \leq(n-1)$, which is absurd. Thus $\operatorname{dim} K=(n-1)$. Since $K \subset F i x(\tau)$, then $(n-1) \leq \operatorname{dim} F i x(\tau)$. If $\operatorname{dim} \operatorname{Fix}(\tau)=n$ then $\operatorname{Fix}(\tau)$ will contain a nonempty open subset of $M$, and we will obtain $\tau=i d$ by Theorem 1.7, which is absurd since $G$ is nontrivial. Hence, $\operatorname{dim} \operatorname{Fix}(\tau)=(n-1)$.

\subsection{Strongly reversible periodic flows}

Let $G$ be a nontrivial periodic flow of period $s$ on a connected $n$-manifold $M$, and assume that $G$ is strongly reversible by an involution $\tau$ such that $\operatorname{Fix}(\tau)$ is an $(n-1)$-manifold. In the following Proposition we prove an important property of $G$ that will be used in the rest of the paper saying that every orbit $G(x)$ in $N$ intersects $F i x(\tau)$ in exactly two points $y$ and $h_{\frac{T}{2}}(y)$; where $T$ is the period of $x$.

Proposition 3.7. Let $G$ be a nontrivial periodic flow of period s on a connected $n$-manifold $M$. If $G$ is strongly reversible by an involution $\tau$ such that Fix $(\tau)$ is an $(n-1)$-manifold, then

1. $N \cap \operatorname{Fix}(\tau) \neq \varnothing$.

2. For every $x \in N, G(x)$ is symmetric and intersects Fix $(\tau)$ in exactly two points $y$ and $h_{\frac{T}{2}}(y)$; where $T$ is the period of $x$.

3. $M=G(\stackrel{F}{F} i x(\tau))$.

Proof. (1) If $N \cap F i x(\tau)=\varnothing$ then $F i x(\tau) \subset F$ and $G_{\mid F i x(\tau)}=i d$. By compactness of $G$, we can find a $G$-invariant open subset $U$ of $M$ such that $(F i x(\tau) \cap U)$ divides $U$ into two connected components $U_{1}$ and $U_{2}$. Since $G$ is connected, each of $U_{1}$ and $U_{2}$ is $G$-invariant, then we can extend the restriction $G_{\mid U_{1}}$ by the identity on $U_{2} \cup(F i x(\tau) \cap U)$. The extension group is a compact Lie group that coincides with the identity on a nonempty open subset, then by Theorem 1.7 it must be trivial. So, $G_{\mid U_{1}}=\{i d\}$. It follows that $G$ is trivial, which is a contradiction. Thus $N \cap F i x(\tau) \neq \varnothing$.

(2) By Proposition 3.4.(1), $N$ is connected. So, by Item 1 and ([3], Theorem 1.2.(2)) every orbit $G(x)$ is symmetric, in particular, we have $\tau(x)=h_{t}(x)$ for some real $t$, and by reversibility of $G$ we obtain $h_{\frac{t}{2}}(x)=\tau\left(h_{\frac{t}{2}}(x)\right)$. So, $G(x) \cap F i x(\tau) \neq \varnothing$. Let $a \in G(x) \cap F i x(\tau)$, and assume that $b$ is another point in $G(x) \cap F i x(\tau)$, then $b=h_{t}(a)$ for some real $t$. Let $T$ denote the period of $a$, then $2 t=n T$ for some integer $n$. The precedent equality implies that $b=h_{n \frac{T}{2}}(a)$. Then either $a=b$ or $b=h_{\frac{T}{2}}(a)$. We conclude that $G(x) \cap F i x(\tau)=\left\{a, h_{\frac{T}{2}}(a)\right\}$. 
(3) By Item (2), $N \subset G(F i x(\tau))$. By Proposition 3.4.(2), $\bar{H}=M$. Since $H \subset$ $N \subset M$, then $\bar{N}=M$. We will show that $F \subset F i x(\tau)$. Let $x \in F$, then there exists a sequence $\left(x_{n}\right) \subset N$ such that $x_{n} \longrightarrow x$. For every integer $n, x_{n}=h_{t_{n}}\left(x_{n}^{\prime}\right)$ for some $h_{t_{n}} \in G, x_{n}^{\prime} \in$ Fix $(\tau)$. By compactness of $G$, we can assume that $h_{t_{n}} \longrightarrow h_{t} \in G$, then $x_{n}^{\prime} \longrightarrow h_{-t}(x)$. Now, $\tau\left(x_{n}\right)=\tau\left(h_{t_{n}}\left(x_{n}^{\prime}\right)\right)=h_{-t_{n}}\left(x_{n}^{\prime}\right) \longrightarrow h_{-t}\left(h_{-t}(x)\right)=x$ since $x \in F$, on the other hand, $\tau\left(x_{n}\right) \longrightarrow \tau(x)$, so, $\tau(x)=x$. We deduce that $F \subset F i x(\tau)$ and so $M=G(F i x(\tau))$.

Lemma 3.8. Let $G$ be a nontrivial periodic flow of period $s$ on a connected $n$-manifold $M$, which is strongly reversible by an involution $\tau$ such that Fix $(\tau)$ is an (n-1)-manifold. Assume that there exist three subsets $A, B$, and $C$ of Fix $(\tau)$ satisfying the following conditions:

1. $C$ is closed in Fix $(\tau)$ and $C \subset$ Fix $\left(h_{\frac{s}{2}}\right)$.

2. $A \cap B=A \cap C=B \cap C=\varnothing$.

3. $A \neq \varnothing$, and $A$ is open and closed in Fix $(\tau) \backslash C$,

4. $B \neq \varnothing$, and Fix $(\tau)=A \cup B \cup C$.

Then $h_{\frac{s}{2}}(A) \neq A$.

Proof. By Proposition 3.7.(3), $M=G(F i x(\tau))$. We will show that $h_{\frac{s}{2}}(A) \neq A$ by contradiction. Assume that $h_{\frac{s}{2}}(A)=A$. Since $A$ is closed in Fix $(\tau) \backslash C$, then $A \cup C$ is closed in Fix $(\tau)$. Which implies that $G(A \cup C)$ is closed in $M$. By conditions (2) and (3), $G(B \cup C)$ is also closed in $M$. Now, we define a map $f$ on $M$ as follows:

$$
\begin{aligned}
f: M & \longrightarrow \begin{cases}h_{\frac{s}{2}}(y), & \text { if } y \in G(A \cup C) ; \\
y, & \text { if } y \in G(B \cup C) .\end{cases} \\
y & \longmapsto
\end{aligned}
$$

We will show that $f$ is a homeomorphism. It suffices to show that if $y \in G(A \cup$ $C) \cap G(B \cup C)$, then $h_{\frac{s}{2}}(y)=y$. If $y \in G(A) \cap G(B)$, then $y=h_{t}(x)=h_{t^{\prime}}\left(x^{\prime}\right)$ for some $x \in A, x^{\prime} \in B$, and $0 \leq t, t^{\prime}<T$, where $T$ is the period of $y$. Since $G(x) \cap F i x(\tau)=$ $\left\{x, h_{\frac{T}{2}}(x)\right\}$ and $A \cap B=\varnothing$, then $x^{\prime}=h_{\frac{T}{2}}(x)$. On the other hand, $h_{\frac{s}{2}}(x) \in F i x(\tau) \cap$ $G(x)$, then either $h_{\frac{s}{2}}(x)=x$ or $h_{\frac{s}{2}}(x) \stackrel{2}{=} h_{\frac{T}{2}}(x) \in B$. Since $h_{\frac{s}{2}}(A)=A$, then $h_{\frac{s}{2}}(x)=$ $x$, which implies that $h_{\frac{s}{2}}(y)=y$. Now, if $y \in G(B) \cap G(C)$ or $y \in G(A) \cap G(C)$, or $y \in G(C)$, then $y=h_{t}(x)$ for some $x \in C$. Since $C \subset F i x\left(h_{\frac{s}{2}}\right)$, then $h_{\frac{s}{2}}(y)=y$. We conclude that $f$ is a homeomorphism. Moreover, $f^{2}=i d$. and $f=i d$ on $G(B \cup C)$. Since $A \cup C$ is closed in Fix $(\tau)$, then $A \cup C$ is closed in $M$, and since the map $\pi: M \longrightarrow M / G$ is continuous and open, then $\pi(A \cup C)$ is closed in $M / G$. Which implies that $\pi(F i x(\tau)) \backslash \pi(A \cup C)=\pi(B)$ is open in $M / G$, and so $\pi^{-1}(\pi(B))=G(B)$ is open in $M$. In the same way, $G(A)$ is open in $M$. Since $B \neq \varnothing$, then $G(B)$ is a nonempty open subset on which $f=i d$, then by Theorem 1.7, $f=i d$. Which implies that $h_{\frac{s}{2}}=i d$ on $G(A \cup C)$ which contains a nonempty open subset of $M$, then 
$h_{\frac{s}{2}}=i d$ on $M$, which contradicts the fact that $G$ is of period $s$. We conclude that $h_{\frac{s}{2}}(A) \neq A$.

Lemma 3.9. Let $G$ be a nontrivial periodic flow of period s on a connected $n$-manifold $M$, which is strongly reversible by an involution $\tau$ such that Fix $(\tau)$ is an (n-1)-manifold. Assume that there exists a closed subset $C$ in Fix $(\tau)$ such that $C \subset F i x\left(h_{\frac{s}{2}}\right)$ and Fix $(\tau) \backslash C$ is nonconnected, then $C$ divides Fix $(\tau)$ into two connected components $A$ and $h_{\frac{s}{2}}(A)$, and $M=G(A \cup C)$.

Proof. Let $A$ be a connected component in Fix $(\tau) \backslash C$. Since $h_{\frac{s}{2}}(F i x(\tau))=$ Fix $(\tau)$, then $h_{\frac{s}{2}}(A)$ is a connected component in Fix $(\tau) \backslash C$. Since $F i x(\tau) \backslash C$ is nonconnected, then $B=F i x(\tau) \backslash(A \cup C) \neq \varnothing$ and $F i x(\tau)=A \cup B \cup C$; where $A, B$, and $C$ satisfy the conditions of Lemma 3.8, then by Lemma 3.8, $h_{\frac{s}{2}}(A) \neq A$. Let $A^{\prime}=$ $A \cup h_{\frac{s}{2}}(A)$. Assume that $F i x(\tau) \neq A^{\prime} \cup C$. Then $B^{\prime}=F i x(\tau) \backslash\left(A^{\prime} \cup C\right)$ is a nonempty subset in Fix $(\tau)$ and $F i x(\tau)=A^{\prime} \cup B^{\prime} \cup C$; where $A^{\prime}, B^{\prime}$, and $C$ satisfy the conditions of Lemma 3.8, then $h_{\frac{s}{2}}\left(A^{\prime}\right) \neq A^{\prime}$; which is a contradiction since $h_{\frac{s}{2}}\left(A^{\prime}\right)=A^{\prime}$. Therefore $F i x(\tau)=A \cup h_{\frac{s}{2}}(A) \cup C$; that is, $C$ divides $F i x(\tau)$ into two connected components $A$ and $h_{\frac{s}{2}}(A)$. Moreover, we have $M=G(A \cup C)$ since $M=G(F i x(\tau))$.

\subsection{An equivalent condition to the existence of a global section}

Using strong reversibility, we give an equivalent condition to the existence of a global section which is an $(n-1)$-manifold by proving Theorem 1.3.

Proof of Theorem 1.3. The implication (1) $\Longrightarrow(2)$ follows from Proposition 3.6.

$(2) \Longrightarrow(1)$. Clearly, $A$ is open in $A \cup F$, then $A$ is an $(n-1)$-manifold, in the same way $B$ is an $(n-1)$-manifold, then $A \cup B$ is an $(n-1)$-manifold. By Proposition 3.4.(1), $M \backslash F$ is a connected open submanifold of $M$ and $G_{\mid M \backslash F}$ is strongly reversible by the involution $\tau_{\mid M \backslash F}$. Since $F i x\left(\tau_{\mid M \backslash F}\right)=F i x(\tau) \backslash F=A \cup B$ is an $(n-1)$-manifold and is nonconnected, then $G_{\mid M \backslash F}$ satisfy the conditions of Lemma 3.9 with $C=\varnothing$, therefore by Lemma $3.9, B=h_{\frac{s}{2}}(A)$ and by Proposition 3.7, $M=G(F i x(\tau))=G(A \cup F)$. It remains to show that every orbit intersects $(A \cup F)$ in exactly one point.

Assume that there exists $y \in M \backslash F$ such that $G(y)$ intersects $A$ in two points $a$ and $b$. Since $B=h_{\frac{s}{2}}(A)$, we will have $a, b$ and $h_{\frac{s}{2}}(a)$ three distinct points in $G(y) \cap F i x(\tau)$ which contradicts the fact that every orbit $G(y)$ in $M \backslash F$ intersects Fix $(\tau)$ in exactly two points (Proposition 3.7.(2)). Therefore $A \cup F$ is a global section for $G$. 


\subsection{Periodic flows that are strongly reversible by reflections have global sections}

Let $G$ be a periodic flow on a connected $n$-manifold which is strongly reversible by a reflection. In this subsection, we will show that $G$ has a global section by proving Theorem 1.4. We begin by proving two important properties of $G$ in the following theorem.

Theorem 3.10. Let $G$ be a nontrivial periodic flow of period $s$ on a connected $n$-manifold $M$. If $G$ is strongly reversible by a reflection $R$, then the following hold.

1. Fix $\left(h_{\frac{s}{2}}\right)=F$.

2. $F$ divides Fix $(R)$ into two connected components $A$ and $h_{\frac{s}{2}}(A)$.

Proof. (1) By Definition 1.2, Fix $(R)$ divides $M$ into two connected components $E$ and $E^{\prime}$; that is, $M \backslash F i x(R)=E \cup E^{\prime}$; where each of $E$ and $E^{\prime}$ is connected, open, and closed in $M \backslash F i x(R)$ and $M$ is the disjoint union $M=E \cup E^{\prime} \cup F i x(R)$. For every integer $k \geq 2$, we have $h_{\frac{-s}{2^{k}}}(M)=M=h_{\frac{-s}{2^{k}}}(E) \cup h_{\frac{-s}{2^{k}}}\left(E^{\prime}\right) \cup h_{\frac{-s}{2^{k}}}(F i x(R))$. It follows that $\operatorname{Fix}(R)=W_{k} \cup W_{k}^{\prime} \cup L_{k}$; where

$$
W_{k}=h_{\frac{-s}{2^{k}}}(E) \cap F i x(R), \quad W_{k}^{\prime}=h_{\frac{-s}{2^{k}}}\left(E^{\prime}\right) \cap F i x(R), \quad L_{k}=h_{\frac{-s}{2^{k}}}(F i x(R)) \cap F i x(R) .
$$

Since $G$ is of period $s, h_{\frac{s}{2}}(F i x(R))=F i x(R)$.

We will show that $L_{k} \subset F i x\left(h_{\frac{s}{2}}\right)$. Let $x \in L_{k}$. Then $R(x)=x$ and $R\left(h_{\frac{s}{2^{k}}}(x)\right)=$ $h_{\frac{s}{2^{k}}}(x)$ which implies that

$$
h_{\frac{s}{2^{k-1}}}(x)=x . \quad(*)
$$

Then $h_{\frac{s}{2}}(x)=\left(h_{\frac{s}{2^{k-1}}}\right)^{2^{k-2}}(x)=x$.

Now, we will show that $h_{\frac{s}{2}}(E)=E^{\prime}$. If not, assume that $h_{\frac{s}{2}}(E)=E$. For $k=2$, we have

$$
F i x(R)=\left(h_{\frac{-s}{4}}(E) \cap F i x(R)\right) \cup\left(h_{\frac{-s}{4}}\left(E^{\prime}\right) \cap F i x(R)\right) \cup\left(h_{\frac{-s}{4}}(F i x(R)) \cap F i x(R)\right) .
$$

Since $G$ is strongly reversible by $R$, and $G$ is nontrivial, then $R(E)=E^{\prime}$, so $h_{\frac{-s}{4}}(E)=$ $h_{\frac{s}{4}}(E)=R h_{\frac{-s}{4}}\left(E^{\prime}\right)$. Then $R\left(W_{2}^{\prime}\right)=W_{2}$. It follows that each of $W_{2}$ and $W_{2}^{\prime}$ is nonempty; otherwise, Fix $(R)=L_{2} \subset F i x\left(h_{\frac{s}{2}}\right)$, and by Theorem 1.7 we will obtain $h_{\frac{s}{2}}=i d$, which is absurd since $G$ is of period $s$. Moreover, $W_{2}$ is open and closed in $F i x(R) \backslash L_{2}$ since $h_{\frac{-s}{4}}(E)$ is open and closed in $M \backslash h_{\frac{-s}{4}}(F i x(R))$. So, $W_{2}, W_{2}^{\prime}$, and $L_{2}$ satisfy the conditions of Lemma 3.8 , thus $h_{\frac{s}{2}}\left(W_{2}\right) \neq W_{2}$. On the other hand, the hypothesis $h_{\frac{s}{2}}(E)=E$ implies that $h_{\frac{s}{2}}\left(W_{2}\right)=W_{2}$, which is a contradiction. Therefore $h_{\frac{s}{2}}(E)=E^{\prime}$, and $h_{\frac{s}{2}}\left(W_{k}\right)=W_{k}^{\prime}$

Now, we will show that $\operatorname{Fix}\left(h_{\frac{s}{2}}\right)=F$. First, we show that for every $k \geq 2$,

$$
L_{k}=F i x\left(h_{\frac{s}{2^{k-1}}}\right) \cap F i x(R)=F i x\left(h_{\frac{s}{2}}\right) \cap F i x(R) . \quad(* *)
$$


Let $y \in L_{k}=h_{\frac{-s}{2^{k}}}(F i x(R)) \cap F i x(R)$, then $y \in F i x\left(h_{\frac{s}{2^{k-1}}}\right) \cap F i x(R)$ (by equality $(*)$ ). Therefore $\quad L_{k} \subset F i x\left(h_{\frac{s}{2^{k-1}}}\right) \cap F i x(R) \subset F i x\left(h_{\frac{s}{2}}\right) \cap F i x(R)$. Conversely, let $y \in$ Fix $\left(h_{\frac{s}{2}}\right) \cap F i x(R)$. If $y \notin L_{k}$, then $y \in F i x(R) \backslash L_{k}=W_{k} \cup W_{k}^{\prime}$. If $y \in W_{k}$, then $h_{\frac{s}{2}}(y) \in$ $W_{k}^{\prime}$, but $h_{\frac{s}{2}}(y)=y \in W_{k} \cap W_{k}^{\prime}=\varnothing$, which is impossible, in the same way $y \notin W_{k}^{\prime}$. Thus $y \in L_{k}$ and Fix $\left(h_{\frac{s}{2}}\right) \cap F i x(R) \subset L_{k}$. We deduce that $(* *)$ is true.

Let $y \in F i x\left(h_{\frac{s}{2}}\right) \cap F i x(R)$, then $h_{\frac{s}{2^{k-1}}}(y)=y$ for all $k \geq 2$. Since $\frac{s}{2^{k-1}} \longrightarrow 0$ when $k \longrightarrow+\infty$, then by Proposition 3.1.(2), y is stationary. Hence, Fix $\left(h_{\frac{s}{2}}\right) \cap F i x(R) \subset$ $F$. Conversely, it is easy to see that $F \subset F i x\left(h_{\frac{s}{2}}\right) \cap F i x(R)$. Therefore, Fix $\left(h_{\frac{s}{2}}\right) \cap$ $F i x(R)=F$. Since Fix $\left(h_{\frac{s}{2}}\right)=G\left(F i x(R) \cap F i x\left(h_{\frac{s}{2}}\right)\right)$, then $F i x\left(h_{\frac{s}{2}}\right)=F$.

(2) By $(* *), L_{k}=F$ for every $k \geq 2$. Then for $k=2, F i x(R)=W_{2} \cup W_{2}^{\prime} \cup F$; where $W_{2}^{\prime}=h_{\frac{s}{2}}\left(W_{2}\right)$, and each of $W_{2}$ and $W_{2}^{\prime}$ is open and closed in Fix $(R) \backslash F$. Moreover, $W_{2} \neq \varnothing$; because if not, $F i x(R)=F$ and $G$ will be trivial, which is a contradiction. Then Fix $(R) \backslash F$ is nonconnected; otherwise $F i x(R) \backslash F=W_{2}=W_{2}^{\prime}$, which is absurd since $W_{2} \cap W_{2}^{\prime}=\varnothing$. By Lemma 3.9, F divides Fix $(R)$ into two connected components $A$ and $h_{\frac{s}{2}}(A)$; where $A=W_{2}$.

Proof of Theorem 1.4. By Theorem 3.10, Fix $(R)=A \cup h_{\frac{s}{2}}(A) \cup F$; where $A$ is open in Fix $(R) \backslash F$. Then $h_{\frac{s}{2}}(A)$ is open in Fix $(R)$, and $(A \cup F)$ is closed in Fix $(R)$. Moreover, $M=G(A \cup F)$. It remains to show that every orbit intersects $(A \cup F)$ in exactly one point. If not, assume that there exists $y \in M \backslash F$ such that there exist $a \neq b \in G(y) \cap A$, then $h_{\frac{s}{2}}(a) \in G(y) \cap h_{\frac{s}{2}}(A)$ and $h_{\frac{s}{2}}(a) \neq a, h_{\frac{s}{2}}(a) \neq b$ since $A \cap h_{\frac{s}{2}}(A)=$ $\varnothing$; which is impossible since every orbit intersects $F i x(R)$ in exactly two points (Proposition 3.7). We conclude that $(A \cup F)$ is a global section for $G$.

We end the paper by an example of a nonperiodic flow which is strongly reversible by a reflection but has no global section.

Example 3.11. Let $G=\left\{h_{t}: t \in \mathbb{R}\right\}$ be the flow on the real line $\mathbb{R}$ defined by

$$
h_{t}(x)= \begin{cases}2^{t} x, & \text { if } x \geq 0 \\ 2^{-t} x, & \text { if } x \leq 0 .\end{cases}
$$

Then, one can easily see that $G$ is strongly reversible by the reflection $s: x \longmapsto-x$, moreover it is nonperiodic and with no global section.

\section{References}

1. Aarts, J. M. and Oversteegen, L. G., Flowbox manifolds, Trans. Amer. Math. Soc. 327 (1991), 449-463. MR1042286

2. Bebutov, M., On the representation of trajectories of a dynamical systems as a family parallel lines, Russ. Bull. Math. Univ. Moscow 2 (1939), 1-22 (in Russian). MR0001679 
3. Ben Rejeb, K., Strongly reversible flows on connected manifolds. (Submitted).

4. Birkhoff, G. D., The restricted problem of three bodies, Rend. Circ. Mat. Palermo 39 (1915), 265-334.

5. Bredon, G. E., Introduction to Compact Transformation Groups, Academic Press, New York, 1972. MR0413144

6. Bhatia, N. P. and Szego, G. P., Stability Theory of Dynamical Systems, Springer, Berlin, 1970. MR0289890

7. Devaney, R. L., Reversible diffeomorphisms and flows, Trans. Amer. Math. Soc. 218 (1976), 89-113. MR0402815

8. Devaney, R. L., Blue sky catastrophes in reversible and Hamiltonian systems, Indiana Univ. Math. J. 26 (1977), 247-263. MR0431274

9. Hofmann, K. H. and Morris, S. A., The Structure of Compact groups: A Primer for the Student - A Handbook for the Expert, De Gruyter Studies in Mathematics 25, de Gruyter, Berlin, New York, 1998, xvii+835pp. MR1646190

10. Halmos, P. R. and Neumann, J. V., Operator methods in classical mechanics, Ann. of Math. 43 (1942), 332-350. MR0006617

11. Kulesza, J., The dimension of products of complete separable metric spaces, Fund. Math. 135 (1990), 49-54. MR1074648

12. Kwun, K. W., Factors of M-space, Michigan Math. J. 9 (1962), 207-211. MR0151946

13. Lamb, J. S. W., Reversing symmetries in dynamical systems, J. Phys. A 25 (1992), 925-937. MR1151093

14. Ikegami, G., On classification of dynamical systems with cross-sections, Osaka J. Math. 6 (1969), 419-433. MR0266224

15. Mostow, G. D., Equivariant embedding in Euclidean spaces, Ann. of Math. 65 (1957), 432-446. MR0087037

16. Montgomery, D. and Zippin, L., Topological Transformation Groups, Interscience, New York, 1955. MR0073104

17. Neumann, D. A., Dynamical systems with cross-sections, Proc. Amer. Math. Soc. 56 (1976), 339-344. MR0407903

18. Sachs, R. G., The Physics of Time Reversal, II, University of Chicago Press, Chicago, 1987. MR0909292

19. Wijsman, R. A., Existence of local cross-sections in linear cartan G-spaces under the action of noncompact groups, Proc. Amer. Math. Soc. 17 (1965), 295301. MR0207892

Khadija Ben Rejeb

Institute of Computer Science and Com-

munication Techniques of Hammam Sousse

University of Sousse

TN-4011 Hammam Sousse

Tunisia

khadija.benrejeb@isitc.u-sousse.tn

Received February 23, 2019

in revised form July 14, 2019 